\title{
Debating the Role of Smartphones and Mobile Applications in Medical Education
}

\author{
C Kim* \\ St Joseph Health Centre, University of Toronto, Canada \\ *Corresponding author: C Kim, St Joseph Health Centre, University of Toronto, Toronto, Canada
}

\section{ARTICLE INFO}

Received: March 17, 2020

Published: April 03, 2020

Citation: C Kim. Debating the Role of Smartphones and Mobile Applications in Medical Education. Biomed J Sci \& Tech Res 26(5)-2020. BJSTR. MS.ID.004420.

\begin{abstract}
Mobile devices have become pervasive within our society. From telecommunications to social media to professional networking platforms, mobile devices are considered a necessity by their users. With the rapid pace of technology innovation and the general evolution of medicine, it would be expected that digital learning platforms, including mobile devices, have also entered the field of medical education. The literature supports the use of mobile devices and medical mobile applications, as a supplement to traditional educational modalities, facilitating access to online medical textbooks, webcasts/podcasts, and online asynchronous classroom. These technologies have the potential of enabling learner-centered and situational learning. However, despite reported benefits there are still concerns that mobile applications focus on lower levels of learning, such as knowledge attainment, with little benefit towards higher levels of Bloom's taxonomy, such as critical thinking. Additionally, only a small percentage of the mobile applications are regulated or accredited by governmental organizations or medical associations, which underscores the concerns regarding content quality and acceptance of its use in medical education. To address these concerns, the following paper will review and highlight the benefits and risks of mobile devices and medical applications as educational tools in medical education.
\end{abstract}

\section{Introduction}

Modern medical education has evolved to incorporate technology and the internet into their learning curriculum as electronic learning (or e-learning). The electronic learning platforms have ranged from personal computers to portable mobile devices, including tablets and smartphones. Mobile devices have revolutionized access to all types of information and data; enabling real-time and on-demand personalized communications; multimedia touchscreen displays operating at increasing speeds and with multi-functionality; delivery of data services; and providing a computing power to document and file. Purposespecific software applications, otherwise known as "apps", have been developed to run on either computers or smartphones or both, and their use is ubiquitous. Medical and healthcare professionals have grown to depend on many applications in their daily personal lives, professional environments, and in patient-care management. Paralleling the rapid rate of health technology development, medical education has likewise evolved over the past several decades, and specific downloadable medical mobile applications can provide medical students and residents opportunities to "learn anywhere and anytime" [1]. Numerous reviews have outlined various categories of medical or healthcare smartphone applications, including applications for scheduling and communication, electronic medical textbook applications, electronic health record applications, and patient-management applications in the form of diagnostic algorithms, clinical-decision support tools, medical calculators and drug compendiums [1-9] (Table 1). 
Table 1: Categories of mobile medical Applications [Data for Table from 5-9].

\begin{tabular}{|c|c|c|}
\hline Category of $\mathbf{m}$ Health applications & How it is used & Examples of apps \\
\hline Information and time management & $\begin{array}{l}\text { Scheduling apps, often used by residents and } \\
\text { faculty for clinical and lecture schedules }\end{array}$ & iCal, Google Calendars \\
\hline Communications by emailing and texting & $\begin{array}{l}\text { Facilitates communication amongst students, } \\
\text { residents, faculty and allied healthcare } \\
\text { practitioners }\end{array}$ & WhatsApp, I Message \\
\hline $\begin{array}{l}\text { Electronic Health Records and Archival } \\
\text { Platforms }\end{array}$ & $\begin{array}{l}\text { Facilitates management and storage of patient } \\
\text { medical records }\end{array}$ & $\begin{array}{l}\text { Electronic Patient Health Record Digital Picture } \\
\text { Archiving \& Communication Systems (PACS) }\end{array}$ \\
\hline Patient management and monitoring & $\begin{array}{l}\text { Applications that assist clinicians to guide } \\
\text { patient care, including diabetes management } \\
\text { and drug-taking reminders. }\end{array}$ & $\begin{array}{c}\text { Contour Diabetes } \mathbb{C} \text { (Ascensia Diabetes), Pfizer } \\
\text { Meds Canada }{ }^{\circledR}\end{array}$ \\
\hline Electronic Textbooks & $\begin{array}{l}\text { Previously hardcopy medical textbooks } \\
\text { commonly used in medical education can now } \\
\text { be available as applications }\end{array}$ & $\begin{array}{l}\text { Harrison's Manual of Medicine (19th Ed), } \\
\text { Uptodate, Medscape }\end{array}$ \\
\hline Drug-referencing tools & $\begin{array}{l}\text { Drug reference compendiums (ex. Compendium } \\
\text { of Pharmaceuticals \& Specialties) are now } \\
\text { replaced by applications }\end{array}$ & Eprocrates, Lexicomp \\
\hline $\begin{array}{l}\text { Clinical Decision-making tools and } \\
\text { Prognostication tools }\end{array}$ & $\begin{array}{l}\text { Applications which enable physicians for } \\
\text { diagnostic differentials \& prognostication }\end{array}$ & MD Calc, QxMd \\
\hline
\end{tabular}

As the integration of smartphones and healthcare-related applications continues within the medical field and subsequently increases within medical education and clinical practice, a debate amongst educators and clinicians remains as to whether students and residents are benefiting from these devices and the associated applications [10-13]. Fundamentally, medical educators need to ask if there is evidence to support the assumption that smartphone usage will improve clinical competency, improve the practice of evidenced-based medicine, reduce medical errors, and in turn, improve patient outcomes. In addition to evaluating the educational advantages, it is imperative that educators, students, learners, clinicians and ultimately professional associations and regulators be aware of the disadvantages and any potential pitfalls from their adoption and usage in medical training.

\section{Medical Mobile Applications as an Educational Supplement rather than an Educational Source}

Medical education has evolved over the past decades from traditional in-person classroom lectures, textbooks and apprenticeship models, founded on behaviorist learning theories [14-17], towards a more learner-centered constructivist approach with an emphasis on competency-based learning, situational learning and critical thinking [9,13,16,17-20]. Modern medical education is now digitalized and mobile devices and applications can support both a situational-learning environment and constructivist-learning by facilitating application of the learned information to clinical practice [16,17]. Mobile devices and medical applications confer a number of benefits within the clinical sphere and medical education. However, it needs to be addressed that mobile applications should be a used as an educational supplement rather than an educational source. One of the major motivating factors for using mobile medical applications is the easy accessibility to large amounts of information. Medical professionals in training, including medical students and residents, use these software applications to enhance their learning, skill development and medical competence. Students are able to carry clinical resources on their person in a convenient, compact and searchable package, which functions as a portable electronic library [8]. Using these resources, medical applications provide an opportunity for learners to participate in their learning process by accessing explicit information and applying it to clinical situations, thereby enabling more effective learning and retention of knowledge experiences, ultimately resulting in improved patient care $[4,10,21]$.

Additionally, trainees report their preference to this learning platform over computers and textbooks, as they provided faster access to information, resulting in less delays during clinical ward rounds and more confidence in collaborating with staff and patients [1,6,11,22-24]. Students and resident trainees report using their mobile applications 39\% of the time in the clinical environment and $51 \%$ of the time for educational purposes [3]. Studies have consistently demonstrated that mobile applications can improve learning outcomes. Systematic reviews, which have included health science students and medical residents, have demonstrated superior test scores in the range of 8-15\%, amongst learners who supplemented their learning with mobile applications compared to those who only used traditional learning methods $[9,13,21,25]$. A concern voiced by educators includes the potential that when learners access information rapidly it may inhibit a deeper internalization of knowledge, a key component of the knowledge acquisition $[6,12,26]$. However, the studies reporting improved test scores did not consider mobile applications as a sole educational resource, but rather as a supplemental one $[6,9,21,25,27]$. In fact, it could be argued that mobile and electronic learning provides additional opportunities to learn while practicing evidence-based medicine, thereby integrating the knowledge and skills attained and transposing them into clinical practice, a crucial component of both physician competency and patient care $[23,28]$. 
In a review of seven randomized trials of healthcare professionals using mobile devices, those clinicians who used their mobile devices in accessing medical information reported better decision-making and better retention of information, when compared to peers who used the equivalent paper resources $[17,29]$. By providing opportunities to practice evidence-based medicine at the patient's bedside, trainees can develop their higher levels of learning including, critical thinking and problemsolving abilities. In order to practice evidence-based medicine and incorporate medical applications in its fold, it is imperative that there is app-literacy amongst learners and clinicians. Reports include a range of $85-91 \%$ of medical students and residents using their mobile devices and medical apps on a daily basis, compared to $10 \%$ of learners using hardcopy textbooks and $20 \%$ using electronic textbooks via a desktop computer [10,30]. Yet, an average of $47 \%$ of surveyed participants, students and residents alike, report difficulty in finding reliable and accurate learning resources, a finding echoed in other studies [3,6,26]. As mobile medical applications are increasingly being used in clinical practice, medical students and residents, in addition to other healthcare practitioners, will need to critically appraise the content presented in the medical applications as 'evidence-based', and thus possibly, useful in clinical practice.

This skill of critically appraising medical applications as they are being used concurrently in clinical training may result in the issue of a hidden curriculum within an EBM focused medical training program and will require the support of academic and medical institutions [31-34]. Recognizing that the present and the future of medical education will involve the integration of mobile devices and medical applications into their undergraduate curriculums and their postgraduate training programs, numerous medical schools have been increasingly supportive of their use by their learners $[21,22]$. As greater involvement from universities, postgraduate education programs, medical associations and professional bodies continue, more advanced applications can be developed, which may further support educational needs, while providing guidelines on their clinical usage.

\section{Cost versus Content Quality of Medical Mobile Applications}

While many medical applications are freely downloadable, others may be associated with an initial subscription fee and further maintenance fees. Considering that the commonly used reference app, Uptodate, has an annual fee of approximately $\$ 520$ US Dollars for an annual subscription [35], this cost may result in inequitable access amongst learners of different socio-economic backgrounds (and countries). In a Canadian study, approximately $38 \%$ of students and residents reported paying for 1-4 medical applications [36] yet another study reported that approximately $43 \%$ of these learners cited cost as a significant limiting factor in acquisition [6]. Medical applications may also be supported through "pushed" advertising, however, there are concerns that applications sponsored by industry may present biased information or have poorer content quality $[21,36,37]$. This is a significant concern as medical and cyber experts estimate that approximately one-third of available medical software applications are developed without the input of appropriate medical professionals [2]. Furthermore, government regulation is still limited as the American Federal Drug Administration (FDA) and Health Canada have only partial and limited oversight of mobile medical applications with particular focus on applications that may be used as medical devices $[2,19,38,39]$. Issues regarding the reliability and accuracy of medical applications are serious concerns and have significant implications for medical error. Mobile applications, if misused, misinterpreted or if the content that is inaccurate or unreliable, can result in erroneous diagnosis and therapeutic management of patients, thereby posing a potential risk to the general public $[39,40,41]$.

As trainees and clinicians increasingly refer and rely on information provided by medical applications it is therefore crucial that the content be accurate, reliable and of the highest quality $[39,40,41]$. Examples regarding poor reliability and inaccurate content have been demonstrated in numerous studies; in a study comparing 23 different opioid conversion apps, inconsistencies and inaccuracies with little concordance between the different opioid conversion calculators were reported [42]; another study comparing 55 marketed pregnancy applications aimed at estimating due dates, reported inaccuracies or no medical references in 71\% [43]; additionally, the well-known example of the dermatology application SkinScan reported a concordance rate of $10.8 \%$ in melanoma diagnosis (a highly malignant skin cancer), when 93 clinical images were compared between the application and references from the National Cancer Institute and Fitzpatrick Dermatology in General Medicine [44]. Ideally, medical applications need to provide reliable, relevant, accurate and up-todate information, while being fiscally accessible for all users, thus avoiding the debate on cost versus content quality. Evaluationtools, including the Health on the Net Foundation (HON), and the Agency for Healthcare Research and Quality (AHRQ) [34], offer a "how to critically appraise" mobile medical applications guide, which consider the content quality, authorship credibility, financial transparency, cost and utility within a clinical context (Table 2). Additionally, online sites, endorsed by professional associations can also recommend unbiased, reliable medical and peer-reviewed applications, including the Medical App Journal and the Journal of Medical Internet Research [2].Recognizing the issue of cost and affordability, medical Institutions, universities and professional associations have also begun to offer endorsed medical applications through their internal information technology programs [2,21,36]. By financially supporting and absorbing the cost of these applications, learners will be able to freely access these reliable and endorsed educational supplements to further support their learning process, without the additional concern of jeopardizing content quality for cost $[21,22]$. 
Table 2: Guide to review a medical application (Data for Table from 2,34,39).

\begin{tabular}{|c|c|}
\hline Point & Questions to Consider \\
\hline Usefulness & Will the content be used in daily practice? \\
\hline Authority and Credibility & $\begin{array}{l}\text { Are the developers qualified and credible? Were medical professionals } \\
\text { involved in the content development? }\end{array}$ \\
\hline Content Accuracy and Relevant & Is the content based on evidence and verifiable? \\
\hline \multicolumn{2}{|l|}{ Is it regularly updated? } \\
\hline Objectivity & Is the content presented fairly balanced with minimal bias? \\
\hline Transparency \& Financial Disclosure & Are there any conflict of interests by the developers? \\
\hline \multicolumn{2}{|l|}{$\begin{array}{l}\text { Are there push advertisements for medications by the same sponsors as } \\
\text { the apps, themselves? }\end{array}$} \\
\hline Design and Functionality & Are the apps 'user-friendly' and functions with minimal technical glitches? \\
\hline Security and Privacy & Will personal data and patient data be protected under federal laws? \\
\hline Ethics & Is the material presented of ethical value? \\
\hline \multicolumn{2}{|l|}{ Are there issues regarding conflict of interests, transparency and privacy? } \\
\hline Cost & $\begin{array}{l}\text { Will the cost of downloading and maintaining the application be of value } \\
\text { in clinical practice? }\end{array}$ \\
\hline
\end{tabular}

\section{Acceptance of the use of Medical Mobile Application's by} Faculty and Patients

Despite the widespread use of smartphones in our daily lives and their incorporation in healthcare and medical education, the acceptability of their use in the classroom and in the clinical setting still remains a hurdle which can negatively impact the learner-teacher and clinician-patient relationship. On one side of the looking glass, patients and teachers may view mobile devices as distractions, potentially resulting in patient resentment and frustration by teachers. Studies have reported that while $75 \%$ of faculty felt that mobile devices are useful educational tools, they were nevertheless a distraction in the classroom, and amongst the faculty surveyed, $46 \%$ felt that mobile devices were also a distraction in the clinical environment [26]. Further to this, a number of surveys have reported patients' and faculty's perceptions of rudeness, laziness and disinterest when smartphones were used in the clinical setting $[1,3,10,22]$. These perceptions often leave students and residents in an unfortunate position; either they do not use these educational supplements or if they do then they feel the need to justify their use $[10,12,22]$. It is important to recognize the negative consequences of these perceptions and how they may negatively impact learning and patient care. Educators and faculty need to become knowledgeable of how medical applications, as with any other technology, are an integral part of medical education. Mobile devices are commonplace and are used at an average rate of $85-87 \%$, amongst medical professionals [24]. It has been established that mobile devices and medical applications provide portability, flexibility and rapid accessibility to large amounts of information enabling healthcare professionals to refer to evidencebased medicine immediately and answer clinical questions during a patient encounter $[11,24]$.

Furthermore, as a learner's medical career matures, there will be differences on how these devices are used, to what frequency and which software applications are being referred to $[6,36,45]$. In general, students will use their devices for note-taking and reviewing online textbooks and lectures, while residents will use their devices for drug-references, clinical calculators and journal articles, and clinicians will refer to subspecialty clinical decision support applications, prognostication clinical calculators and drug references $[1,2,6,24,30,36,45]$. Improved patient-care via the use of mobile applications in the clinical environment can further be evidenced during the transition period as medical student's transition to resident doctors, and as these residents transition to staff physicians [11].As patient care is becoming increasingly complex and providing medical care is still expected to be efficient, safe and of the highest quality, the stakes are high and these new physician residents often feel unprepared when it comes to decision making, and prescribing therapy $[30,37,45]$. In order to provide optimal patient care, physicians will rely on clinical decision support systems and drug reference applications [11,24,46]. Studies have reported that whereas non-smartphone physician users would only search for answers to clinical questions in $6 \%$ of their cases, smartphone owners were successful $95 \%$ of the time when they referred to medical information on their phones $[19,46]$.

When these decision support applications are used, inappropriate investigations are avoided, while appropriate tests and therapies are ordered in a timely manner with an increase in the average rate of appropriate electronic ordering and patient management from $52 \%$ to $64 \%$, and a decrease in medical order error rates from $22 \%$ to $8 \%[3,7,11,19,29]$. As mobile applications, these decision support tools can be readily accessed by residents and clinicians in order to make immediate evidence-based decisions while avoiding harm in potentially stressful situations where critical information retrieval is time-sensitive and crucial $[8,11,47]$. Finally, it is without a doubt that effective and reliable communication amongst healthcare professionals and their patients 
is crucial component of patient management. In this regard, mobile devices have significantly improved communication beyond simple phone calls and text messaging with applications designed for Multimedia Messaging Services (MMS), emails, video-conferencing, and instant peer-to-peer or clinician-patient communication capabilities, thereby improving patient care and outcomes [26]. Communication within medical education has improved as learners and their educators are able to communicate and collaborate through messaging groups, video-conferencing and social media, without the added stress of coordinating different schedules (and locations) for in-person meetings $[26,48]$. Further to this, mobile devices can facilitate communication with remote areas (countries or continents) with limited (or no) access to local specialists, providing equitable medical care on a local and global basis $[8,48]$. Likewise, patient-care has evolved with Electronic Health Records (EHR) being available as mobile secured applications, which has further enabled clinicians to conveniently review and respond to patient-related issues rapidly and remotely. These electronic health records, along with the various communication platforms, enable healthcare providers to communicate rapidly amongst themselves and provide bi-directional physician-patient communication, particularly in the cases of chronic diseases $[8,11,19,28,49,50]$. Mobile devices and medical applications are able to provide learners and clinicians, rapid and reliable educational tools which can be used in a situational context for immediate and improved patient care, as well as provide communication versatility amongst healthcare professionals and with patients. Understanding these roles that medical applications have in medical education and clinical care, how their use are beneficial towards patient-care, will lead to a wider acceptance of mobile devices and medical application use in the clinical environment by educators, faculty and ultimately, patients.

\section{Conclusion}

Over the last 50 years technology has changed our personal and professional lives. The practice of medicine has also rapidly evolved over this period thanks in part to the advancement of science and medicine but also the improvement in communication and the speed of knowledge dissemination. As medicine and technology intertwine and advance together, there is an increasing demand and expectation for accurate, reliable and efficient medical care. To answer these expectations, modern medical education has evolved from traditional behaviorist learning models which included in-person classroom teaching and apprenticeships, towards situational and constructivist learning models, with the incorporation of online classes, web-conferences, simulation learning, virtual-reality learning, podcasts, chat groups and social media, to name just a few. Amongst the e-learning tools, mobile devices and mobile medical software applications are being used by medical students, residents and faculty at an exponential rate. Clinician-educators have expressed concerns that smartphones and mobile applications may serve as 'shortcuts' to effective learning, potentially causing some learner-educator discordance, foster superficial learning, detract from acquiring deeper learning, and undermine a learner's potential for critical thinking and problemsolving skills $[6,13]$.

Upon review of the published literature, these concerns appear to be unfounded and not evidence-based. Medical students and residents are using these technologies as an educational supplement, referring to evidence-based medical information in real-time and within clinical context, thus enhancing their learning activities, while providing efficient, safe and optimal standard of care for their patients. If we consider a situational and constructivist approach to learning, these mobile 'shortcuts' to information can be accessed, while at the patient's bedside and applied in the clinical context immediately, thereby enabling learners to easily integrate the new information into their foundational knowledge immediately, resulting in better retention and new knowledge constructs being formed. Thus, it could be argued that rather than undermine learning, mobile devices and their applications enable residents to 'read around' clinical cases in real time, when retention is the most optimal $[23,24,28,30]$. Furthermore, studies that reported the improved learning outcomes of those students and residents using mobile devices, considered their use as an educational supplementary tools, rather than as educational sources [1,21,24,25,27,45,47]. Furthermore, how these medical applications will be used by students, residents and clinicians will differ at the various stages of their career; students will refer to online textbooks and lectures for information gathering, while residents will more often refer to clinical decision support applications, drug references and prognostication clinical calculators [1,6,24,45]. Thus far the literature has demonstrated that the benefits of mobile devices and medical applications in knowledge and evidence-based clinical training outweigh the educational concerns, it is therefore important that educators not only accept smartphone and medical application use but support their learners using these educational supplements.

The cost and the accessibility of medical applications, the quality and reliability of the medical information provided by the applications will require scrutiny and input from medical professionals. Recognizing that there are some applications of questionable quality, users will need to be aware of which applications were developed with contributions from medical professionals, government-regulated, peer-reviewed and endorsed by institutions and professional bodies [2,42]. As educators and clinicians become skilled at appraising medical applications, it will become crucial to transfer that knowledge to learners, while universities and institutions will need support and provide learners with endorsed, peer-reviewed applications without cost concerns $[2,3,21]$. The future of mobile devices and the widespread acceptance of reliable and accurate medical applications in 
medical education and patient care is tangible. The literature has consistently demonstrated improved educational outcomes, improvements in patient care outcomes and decreases in medical errors. The versatility, accessibility and reliability of mobile devices and the numerous mobile applications will enable equitable and improved patient care. Thus, the beneficial use mobile devices and associated medical software applications can be extended beyond medical education and be applied to patient care, as a whole. It can, therefore, be concluded that the benefits of smartphones and medical applications outweighs their potential pitfalls.

\section{References}

1. Payne KF (2012) Smartphone and Medical-related App use among Medical Students and Junior Doctors in the United Kingdom; a regional study. BMC Med Infor \& Dec. Making 12: 121.

2. Yip Y (2014) There is an App for that: the use of Mobile Medical Applications in ClinicalPractice. Ontario Medical Review, p. 1-4.

3. Ventola C (2014) Mobile Devices and Apps for Health Care Professionals Uses and Benefits.P\&T 39(5): 356-364.

4. Snashall E (2016) The Use of Smartphone Applications in Medica Education. Open Med Jour 3(suppl-3): 322-327.

5. Ozdalga E (2012) The Smartphone in Medicine; a Review of Current and Potential UseAmong Physicians and Students. Jour of Med. Internet Res 14(5): e128.

6. Wallac S (2012) It's On My Iphone: Attitudes to the Use of Mobile Computing Devices inMedical Education. BMJ 2(4): e0001099.

7. Mickan S (2013) Evidence of Effectiveness Healthcare Professionals Using Handheld Computers: A Scoping Review of Systematic Reviews. J Med Int Res 15(10): e212.

8. Boulos M (2014) Mobile Medical and Health Apps: State of the Art, Concerns, Regulatory Control and Certifications. Online Jour. Public Health Infor 5(3): 229.

9. Car J (2019) Digital Education in Health Professions; the need for Overarching Evidence Synthesis. Jour Med Int Res 21(2): e12913.

10. Hardyman W (2013) Mobile Technology Supporting Trainee Doctor's Workplace Learning and Patient Care. BMC Med Ed 13(6): 1-10.

11. Webb K (2016) Can a Mobile App improve the Quality of Patient Care Provided by TraineeDoctors? Analysis of Trainee Reports. BMJ Open 6 (9): e012075.

12. Delgaty L (2017) The Dark Side of Technology in Medical Education Med Ed Pub p. 1-13.

13. Dunleavy G (2019) Mobile Digital Education for Health Professions; Systematic Review andMeta-Analysis by the Digital Health Education Collaboration. Jour of Med Int Res 21(2): e12937.

14. Swing S (2010) Perspectives on Competency-Based Medical Education from the LearningSciences. Med Teach 32(8): 663-668.

15. Mc Gaghie W (2011) Does Simulation-based Medical Education with Deliberate Practice Yield Better Results than Traditional Clinical Education? A Meta-Analytic Comparative Review of the Evidence. Acad. Med. 86(6): 706-711.

16. Yilmaz K (2011) The Cognitive Perspective on Learning: It's Theoretical Underpinnings andImplications for Classroom Practices. The Clearing House 84: 204-211.

17. Al Hamdani (2013) Mobile Learning: A Good Practice. Proceeding Soc. And Behav Sc 103: 665-674.

18. Al Eq A (2010) Simulation-based medical teaching and learning. J Family Community Med 17(1): 35-40.
19. Badawy S (2015) Health-related smartphone Apps: Status Update for Heme Onc Practitioners. The Hematologist 12(4): 13-14.

20. Hsaio C (2016) Using Interactive Multi-media eBooks for Learning Blood cell Morphology in Pediatric Hematology. BMC Med Ed 16(1): 290.

21. Rasmussen K (2014) Offline eLearning for Undergraduate in Health Professions; A Systematic Review of the Impact of Knowledge, Skills, Attitudes and Satisfaction. Jour. Global. Health 4(1): 010405.

22. Ellaway R (2014) Left to their own Devices: Medical learner's use of Mobile Technologies.Med Teacher 36 (2): 130-138.

23. Friedrichs H (2014) Practicing Evidence based medicine at the bedside; a randomizedcontrolled pilot study, in undergraduate medical students assessing the practicality of tablets, smartphone and computers in clinical life. BMC Med Inf and Dec Mal 5(14): 113

24. Patocka C (2018) Point-of-care Resource Use in the Emergency Department: ADevelopmental Model. AEM Ed Train 2(3): 221-228.

25. Low D (2011) A Randomized Control Trial to determine if use of the I Resus Application on a Smartphone Improves the Performance on Advanced Life Support Provide in a Simulated Medical Emergency. Anesthesia 66(4): 255-262.

26. Skiba D (2011) On the Horizon Mobile Devices: Are They a Distraction or another LearningTool? Emerg Tech 32(3): 170-173.

27. Martinez F (2017) Implementation of a Smartphone Application in Medical Education: A Randomized Trial (I START). BMC Med Ed 17(1): 168.

28. Lohnari T (2016) Use of Mobile Application in Healthcare; a Review. Into Jour of Engin. Resand Gen Sc 3: 250.

29. Mickan S (2014) Use of Handheld Computers in Clinical Practice; a Systematic Review. BMC Med Inf \& Dec Mak 14(1): 56

30. Bullock A(2015) How a Mobile Apps Supports the learning and Practice of NewlyQualified Doctors in the UK; an Intervention Study. BMC Med Ed 15: 71-85.

31. Rhodes M (2006) Teaching Evidence-based Medicine to Undergraduate MedicalStudents: A Course Integrating Ethics, Audit, Management and Clinical Epidemiology. Med Teach 28(4): 313-317.

32. Timm DF (2012) Critical Appraisal Process: Step-by-Step. South Med J 105(3): 144-148.

33. Rezaiean M (2013) How to Teach Medical Students to Critically Appraise a Published Article. Jour. Ed and Health Promotion 2: 8.

34. Bozikov J (2017) Training of Students for Critical Evaluation of Mobile Health Applications. MEDINFO 2017: Precision Healthcare through Informatics 245: 1320 .

35. https://store.uptodate.com.

36. Boruff J (2014) Mobile Devices in Medicine: a survey of how Medical Students, Residents and Faculty use Smartphones and Other Mobile Devices to find Information. Med Lib Assoc 102(1): 22-30.

37. Shah S (2016) Hematology on the Move: How are Mobile Health Apps helping Patients? ASH Clinical News.

38. Shuren J (2014) The FDA's Role in the Development of Medical Mobile Applications. Clinical Pharma \& Thera 95(5): 485-458.

39. Sharp M (2017) Mobile Medical Apps and mHealth Devices: A Framework to Build MedicalApps and mHealth Devices in an Ethical Manner and Promote Safer Use; a Literature Review. Infor for Health. European Federation for Medical Informatics (EFMI) and IOS Press. 235: 363-367.

40. Gonnerman A (2015) Draft Guideline for the Development of Evidencebased Medicine-Related Apps. European Fed for Med. Inform 21(1): 1020.

41. Yetisen A (2014) The Regulation of Mobile Medical Applications. Lab on a Chip14(5): 833-840. 
42. O Neil S (2013) Clinical Involvement and Transparency in Medical Apps; Not all Apps are Equal. Colorect Dis 15(1): 122.

43. Chyjek K(2015) Rating Pregnancy Wheel Applications using the APPLICATIONS Scoring System. Obs. \& Gyn 125(6): 1478-1483.

44. FerraroN (2013) Skin Scan: A Demonstration of the need of FDA regulation of MedicalApps on IPhone. J Am Acad Derm 68(3): 515-516.

45. Shenouda (2018) The Toll Of The Smartphone In The Transition From Medical Student To Foundation Trainee; A Qualitative Interview And Focus Group Study. BMC Med Ed 18(1): 175.

46. Ely (2005) Answering Clinical Questions; Obstacles and Potential Solutions. J Am MedInfor Assoc 12(2): 217-224.

47. O Reilly M (2014) DOCSS: Doctors On-call Smartphone Study. Ir J Med Sc. $183(4)$ : 573-577.

ISSN: 2574-1241

DOI: 10.26717/BJSTR.2020.26.004420

C Kim. Biomed J Sci \& Tech Res

(C) This work is licensed under Creative

Submission Link: https://biomedres.us/submit-manuscript.php
48. O Donovan J (2014) Using Low-Cost Android Tablets And Instructional Videos To Teach Clinical Skills To Medical Students In Kenya; A Prospective Study. J Royal So of Med 37: 1-7.

49. Breen S (2015) The Patient Remote Intervention And Symptom Management Systems (PRISMS) A Telehealth- Mediated Intervention Enabling Real-Time Monitoring Of Chemotherapy Side-Effects In Patients With Haematological Malignancies: Study Protocol For A Randomized Controlled Trial. Trials 16(1): 472.

50. Maguire R (2017) The eSmart Study Protocol: A Randomized Controlled Trial To Evaluate Electronic Symptom Management Using The Advanced Symptom Management System (Asyms) Remote Technology For Patients With Cancer. BMJ Open 7(5): e015016.

$\begin{array}{ll}\text { BIOMEDICAL } & \text { Assets of Publishing with us } \\ \text { RESEARCHES } & \text { - Global archiving of articles } \\ \text { - Immediate, unrestricted online access } & \text { - Rigorous Peer Review Process } \\ & \text { - Authors Retain Copyrights }\end{array}$

